\title{
IDN Server Proxy Architecture for Internationalized Domain Name Resolution and Experiences with Providing Web Services
}

\author{
JENG-WEI LIN \\ Tunghai University, Taiwan \\ JAN-MING HO \\ Academia Sinica \\ LI-MING TSENG \\ National Central University \\ and \\ FEIPEI LAI \\ National Taiwan University
}

\begin{abstract}
The composition of traditional domain names are restricted to ASCII letters, digits, and hyphens (abbreviated as LDH). This makes it difficult for many to use their native language to name and access their Internet hosts. The IETF IDN (Internationalized Domain Name) Working Group proposes a mechanism, IDNA (Internationalizing Domain Names in Applications), for internationalized access to multilingual domain names. The proposal uses a preparation process that converts a Unicode IDN into an ACE (ASCII Compatible Encoding) string that uses only LDH. Thus, applications can look up the ACE string by using the existing DNS infrastructure. However, some of the domain name strings embedded in multilingual content do not have any charset tag so they cannot appropriately be converted into ACE strings. We noticed that many Internet applications allow users to use non-ASCII domain names. We were motivated to design an architecture for IDN resolution as well as to minimize the cost of modifying legacy Internet applications. We specifically focus on designing an IDN server proxy, which is located on the domain name server side, to handle domain names in multiple encodings. In this article, we study several architecture design issues including detection of charset encoding, routing of non-ACE IDN lookup requests, and so on.
\end{abstract}

Authors' addresses: Jeng-Wei Lin, Department of Information Management, Tunghai University, 181 Sec. 3, Taichung Harbor Rd., Taichung 40704, Taiwan; email: jwlin@thu.edu.tw; Jan-Ming Ho, Institute of Information Science, Academia Sinica, 128 Sec. 2, Academia Rd., Taipei 115, Taiwan; Li-Ming Tseng, Department of Computer Science and Information Engineering, National Central University, 300 Jhongda Rd., Jhongli City, Taoyuan County 32001, Taiwan; Feipei Lai, Department of Computer Science and Information Engineering and Department of Electronic Engineering, National Taiwan University, 1 Sec. 4, Roosevelt Rd., Taipei 106, Taiwan.

Permission to make digital or hard copies of part or all of this work for personal or classroom use is granted without fee provided that copies are not made or distributed for profit or direct commercial advantage and that copies show this notice on the first page or initial screen of a display along with the full citation. Copyrights for components of this work owned by others than ACM must be honored. Abstracting with credit is permitted. To copy otherwise, to republish, to post on servers, to redistribute to lists, or to use any component of this work in other works requires prior specific permission and/or a fee. Permissions may be requested from Publications Dept., ACM, Inc., 1515 Broadway, New York, NY 10036 USA, fax: +1 (212) 869-0481, or permissions@acm.org.

(C) 2006 ACM 1533-5399/06/0200-0001 $\$ 5.00$ 
With respect to these design issues, we present an IDN server proxy architecture which stores ACE IDNs in domain name servers. Note that traditional domain name servers can be used without modification. An IDN server proxy, called Octopus, is employed on the domain name server side. Octopus converts a non-ACE IDN string into ACE upon receiving an IDN lookup request from remote users or autonomous systems. The ACE string is then forwarded to backend domain name servers (where the traditional domain names and ACE IDNs are stored) for further processing. This allows Internet users to access IDNs without having to upgrade their software.

Based on the design and implementation of Octopus, we deployed a CDN (Chinese domain name) trial in July 2002. In this article, we present the results of testing Octopus IDN lookup functions as well as our experiences in providing CDN Web services. Several types of errors can occur if applications are unable to handle IDNs adequately. For example, a Web browser may erroneously parse an IDN within a URL. Many legacy Web servers are unable to process the IDN of a virtual host. Web application servers may have trouble completing some actions such as redirecting Web pages to alternative Web pages. Our studies help service providers understand potential problems when non-ASCII domain names are used and the best common practice at this stage. As well, the experiences give some guidance for software developers to develop IDNA-compliant Internet applications.

Categories and Subject Descriptors: C.2 [Computer Communication Networks]: Network Protocols-Applications; D.2.11 [Software Engineering]: Software Architectures-Domain-specific architectures

General Terms: Design, Experimentation, Standardization

Additional Key Words and Phrases: Internationalized domain name, DNS server proxy, application server, multilingual

\section{INTRODUCTION}

An increasing number of people want to access the Internet using their native language. However, traditional domain names are restricted to LDH characters (ASCII letters, digits and hyphens) [Mockapetris 1987]. This makes it difficult for people to use their native language to name and access their Internet hosts. Allowing users to access the Internet via multilingual domain names would be a significant improvement in service. In this article, we consider the problems for a service provider in delivering services to users who are not familiar with English. Specifically, we target our studies at domain name service and Web services.

The IETF IDN (Internationalized Domain Name) Working Group proposes a mechanism, IDNA (Internationalizing Domain Names in Applications) [Faltstrom et al. 2003; Hoffman and Blanchet 2003; Costello 2003], for internationalized access to multilingual domain names in Unicode. Figure 1 illustrates name processing in IDNA. The proposal uses a preparation process to convert a Unicode IDN into an ACE (ASCII Compatible Encoding) string that uses only $\mathrm{LDH}$. Once the IDN is registered in an IDN registry, the IDN registry stores the ACE string in the domain name server. When an IDNA-aware application looks for a host using its IDN, the application converts the IDN into an ACE string so that the existing DNS infrastructure can then resolve the ACE string into the host's IP address. However, some of the domain name strings embedded in multilingual content do not have any charset tag so they cannot appropriately be converted into Unicode IDNs and, thus, the ACE strings. Note that IDNA is able to use the existing DNS infrastructure without modifying 


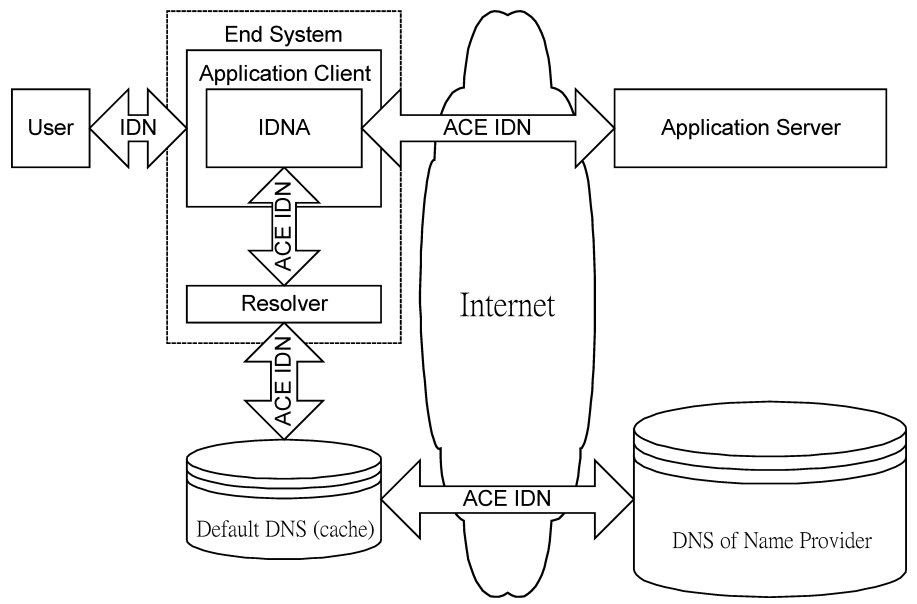

Fig. 1. Name processing in IDNA.

domain name servers. However, it does require that an IDNA-compliant module be integrated into every legacy Internet application in order to process IDNs properly.

We noticed that many legacy Internet applications allow users to use nonASCII domain names. For example, Internet Explorer or Netscape users are able to use Chinese domain names $(\mathrm{CDN})$ to access Internet hosts. Depending on the user's platform, the IDN will either be encoded in its local encoding, in UTF8 [Yergeau, 1998], or in other less commonly used encodings. Consequently, we are motivated to design an IDN service architecture that provides IDN resolution in multiple encodings. As well, we aim to minimize the cost of modifying legacy Internet applications.

Instead of converting IDNs into ACE strings in applications, an ISP can set up an IDN proxy to resolve IDNs. An example of such a proxy is the mdnsproxy which is included in the mDNkit [JPNIC] on the client side to convert non-ACE IDN lookups into ACE. Thus, users can reconfigure the default DNS settings of their platforms to the IDN proxy so that DNS query packets will be sent to the IDN proxy. However, this approach requires all ISPs to set up IDN proxies. Furthermore, the string in the QNAME (query domain name) field of a DNS query packet may represent a traditional Chinese domain name in BIG5 ${ }^{1}$, a simplified Chinese domain name in GB-2312/GBK, a Japanese domain names in Shift-JIS, or an IDN in any possible encoding. The string may represent two or more IDNs in different encodings of different languages ${ }^{2}$, referred to as a binary collision. An IDN proxy must be able to recognize the encoding used to encode the IDN. Since the number of languages and their encodings that users and applications use may be large, IDN proxies have difficulty recognizing a specific encoding among all possible encodings. It is also true that, while IDN

${ }^{1}$ BIG5 is a charset for traditional Chinese. GB-2312 and GBK are two charsets for simplified Chinese. GBK is a backward compatible successor of GB-2312. Shift-JIS is a charset for Japanese. ${ }^{2}$ For example, a binary string 'B9 AB CB BE' (in hexadecimal) represents either 公司 (in GB-2312), 鼠侗(in BIG5), or 个批 (in Shift-JIS). 
services continue to be deployed, the consistency between all IDN proxies set up by different ISPs is hard to maintain.

Unlike IDN proxies, which have to face users or autonomous systems that might access multilingual services in any possible charsets, a service provider can choose the target charsets that are commonly used by their clients. If IDN registries prevent binary collisions during registration, it is possible for application servers to recognize the encoding used to encode an IDN. Note that binary collision prevention will cause the name space to shrink. Service providers can deliver better services by supporting more encodings.

We present a new architecture, IDN server proxy, which provides transparent IDN resolution on the domain name server side. In this article, we refer to a server proxy as a network entity that facilitates a set of servers to enhance their services to the rest of the Internet. This is in contrast to the typical usage of the term proxy which defines a network entity that facilitates the ability of a set of clients to access the rest of the Internet. In this architecture, ACE IDNs are stored into the domain name servers of the name holders. Note that traditional domain name servers can be used without modification. An IDN server proxy, called Octopus, is employed on the domain name server side to facilitate a set of domain name servers by providing non-ACE IDN resolution. Upon receiving a DNS query packet from remote users or autonomous systems, Octopus converts the non-ACE IDN into an ACE string. The ACE string is then forwarded to backend domain name servers (where the traditional domain names and ACE IDNs are stored) for further processing. This allows Internet users to use nonASCII domain names without having to upgrade their software.

Based on the design and implementation of Octopus, we deployed a CDN (Chinese domain name) service trial in July 2002. We study the interoperability of Internet applications when non-ASCII domain names are used and the best common practice at this stage. Experiment results show that Octopus successfully provided IDN resolution in multiple encodings for legacy Internet applications. However, users and service providers still experienced unsuccessful IDN access in the beginning of the CDN services. We studied several types of errors that cause unsuccessful IDN access on the Web such as improper Web server configuration, generic multilingual text processing errors, and so on. Solutions were then developed, including the use of an IDN-aware Web redirection server.

This article is organized as follows. In Section 2, we present issues in designing an IDN server proxy architecture; in Section 3 our system implementation, Octopus, is described. We present our experiences with providing IDN-enabled services and discuss relevant issues in Section 4. And we discuss our conclusion in Section 5.

\section{DESIGNING AN IDN SERVER PROXY}

Currently, an IDN in a DNS query packet will probably be encoded in its local encoding, UTF8, ACE, or other less commonly used encodings, depending on the user's application and platform. Generally, the domain name server will receive query packets that contain IDNs in their original binary representations. Under 


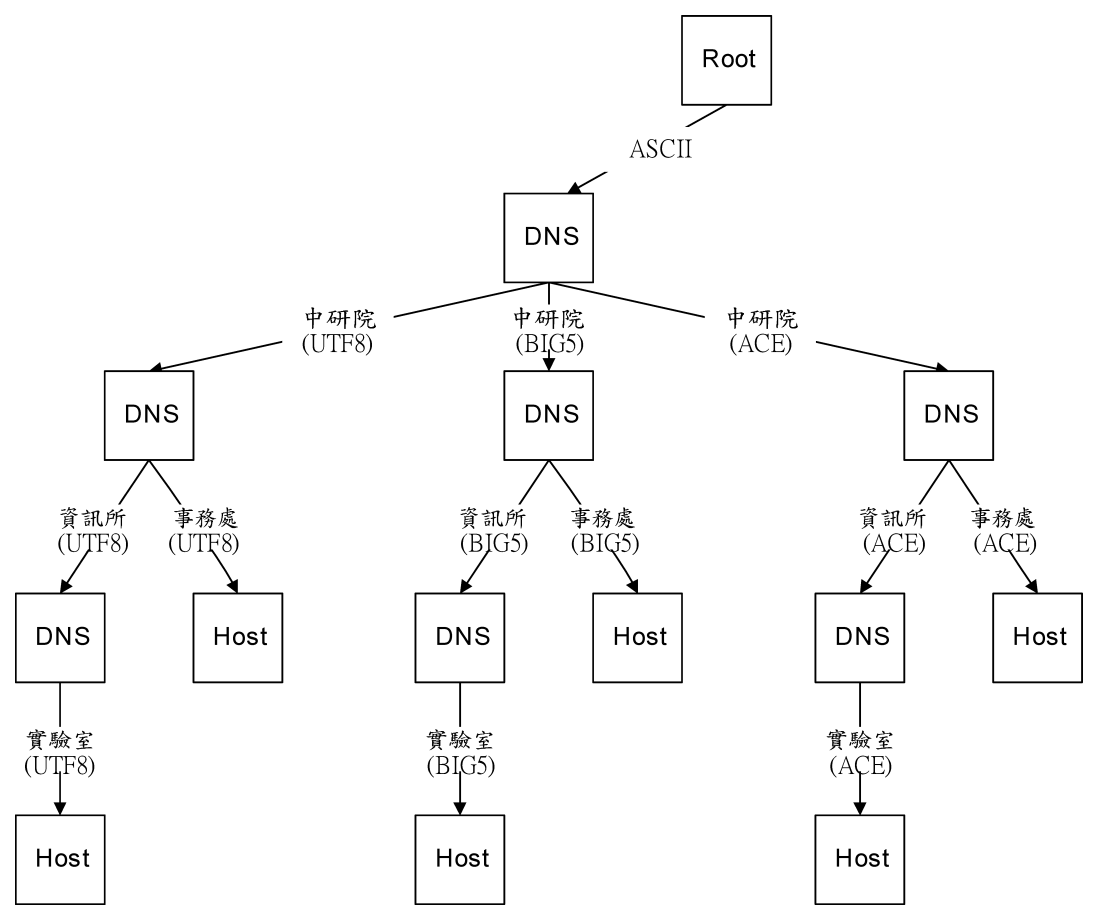

Fig. 2. Maintenance of IDNs in multiple encodings.

some circumstances, however, an IDN might be falsely interpreted, ${ }^{3}$ and the domain name server will receive a vitiated representation of the IDN.

To provide IDN resolution in multiple encodings on the domain name server side, name holders must upgrade their domain name servers to support multiple encodings and maintain IDNs in multiple zone files for each encoding. Figure 2 depicts one example in which Chinese domain names are maintained in BIG5, UTF8, and ACE. However, customized modification to domain name server software is required to support multiple encodings. Maintaining multiple zone files for each encoding is fatigue and error prone. Chung and Leung [2002] proposed using Dynamic-CNAME to dynamically synthesize back subdomains to the query so that a successful delegation can be effectively made.

We try to use a single IDN server proxy to provide non-ACE IDN resolution for a set of domain name servers as shown in Figure 3. Thus, the effort of maintaining IDNs in multiple encodings is significantly downscaled to maintain only ACE IDNs. Name holders as well do not need to upgrade their domain name servers. Acting as a real domain name server, when the IDN server proxy receives a DNS query packet, it first detects the encoding and forward transcodes the IDN into ACE. It then uses the ACE string to retrieve the queried resource records from the domain name server of the ACE IDN subdomain. It backward transcodes relevant IDNs (such as those returned in CNAME, MX, NS resource

${ }^{3}$ Under Windows 98, Linux, and FreeBSD, \(backslash) used in many multiple-byte charset encodings will cause a false escape sequence. 


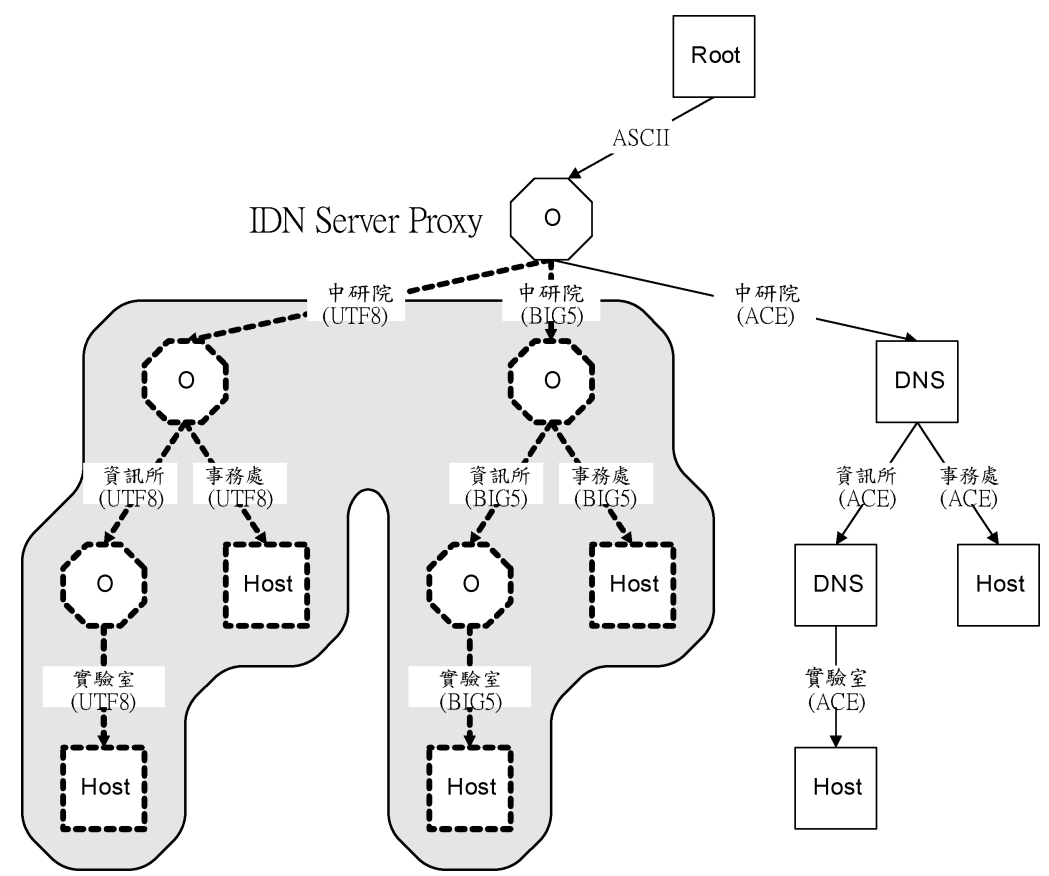

Fig. 3. Provision of name resolution for non-ACE IDNs.

records) from ACE into the original encoding if necessary, and finally returns authoritative answers to the remote user.

\subsection{Issues in Designing an IDN Server Proxy}

To build such a design, several issues were involved which will be described in this section.

2.1.1 Detection of Encoding. A DNS query packet arriving at the DNS server proxy may come from various applications and platforms that encode IDNs in various encodings. Furthermore, a binary collision may occur when the domain name string can represent two or more IDNs in different encodings. Since there is no charset tag in the packet, the IDN server proxy must be able to decide how the IDN is encoded.

2.1.2 Transcoding Non-ACE and ACE Domain Names. Transcoding of an IDN between different charsets will fail if a character of the IDN is not in the target charset. Since ACE is an encoding for Unicode (a superset of most character sets used in electronic information processing), forward transcoding to ACE should always succeed. However in some situations, backward transcoding relevant IDNs from ACE into local encodings might fail. If the queried IDN has a CNAME (a canonical name of an alias) resource record specifying a canonical IDN that uses characters beyond the scope of the character set of the encoding of the DNS query packet, backward transcoding of the canonical IDN will fail. 
The lengths of the same IDN in different encodings may vary. For example, the length of a Chinese domain name label 公司 is 4-octet long in BIG5 and 6octet long in UTF8. Transcoding of an IDN into another encoding may generate a longer string. The total length of a domain name is restricted to 255 octets or less, and the length of a domain name label is restricted to 63 octets or less. After transcoding, a valid IDN in one encoding may become invalid in another encoding. As a result, the IDN server proxy should be able to detect possible errors that may occur when transcoding IDNs between different encodings.

2.1.3 Routing of a Non-ACE IDN Lookup. Conceptually, the domain name system is a single tree-like distributed database and each domain name server has one or more pieces, called zones, of the total tree data. A DNS lookup usually involves a traverse of the DNS tree from the root down to the zone containing the specified resource records. However, there are neither domain name servers set up for non-ACE IDN subdomains nor zone files containing the resource records associated with non-ACE IDNs. As the IDN server proxy is set up to provide transparent resolution to all non-ACE IDN lookups, all non-ACE IDN lookups should be sent to the IDN server proxy.

2.1.4 Inference of Domain Name Caching. Domain name servers usually have caches that enable quick responses to often asked questions. However, the cached information of IDNs in some encodings may cause errors. A generic BIND, the most popular domain name server software in use, falsely matches (a) 大逸 encoded in BIG5 with (b) 大象 also encoded in BIG5 because the former is the case-insensitive equivalent of the latter. If a domain name server has cached resource records for (a), it will return those records as the answer to a query that looks up the same type of resource records for (b). This is a severe detriment to DNS functionality. The IDN server proxy must work to eliminate this type of situation if it is to support encodings such as BIG5.

2.1.5 Availability and Scalability. As all non-ACE IDN lookups are routed to the IDN server proxy, the design of the IDN server proxy should be failtolerant in order to prevent a single point of failure. The IDN server proxy should also be scalable to handle a large number of DNS lookups as they typically involve a traverse of the DNS tree which consumes a lot of computing resources.

\section{OCTOPUS IDN SYSTEM}

Octopus IDN System is a realization of IDN server proxy architecture previously described. In this section, we discuss the design of the Octopus IDN System and its current status.

As shown in Figure 4, the Octopus IDN System consists of an Octopus Mapper and multiple generic domain name servers, $\mathrm{DNS}_{1}, \mathrm{DNS}_{2}$ to $\mathrm{DNS}_{\mathrm{n}}$, which are configured to retrieve resource records from ACE IDN subdomains.

The Octopus Mapper is the main component of the Octopus IDN System. All non-ACE IDN lookups will be routed to the Octopus Mapper. When the Octopus Mapper receives a DNS query packet, it 1 ) detects the encoding of the non-ACE 


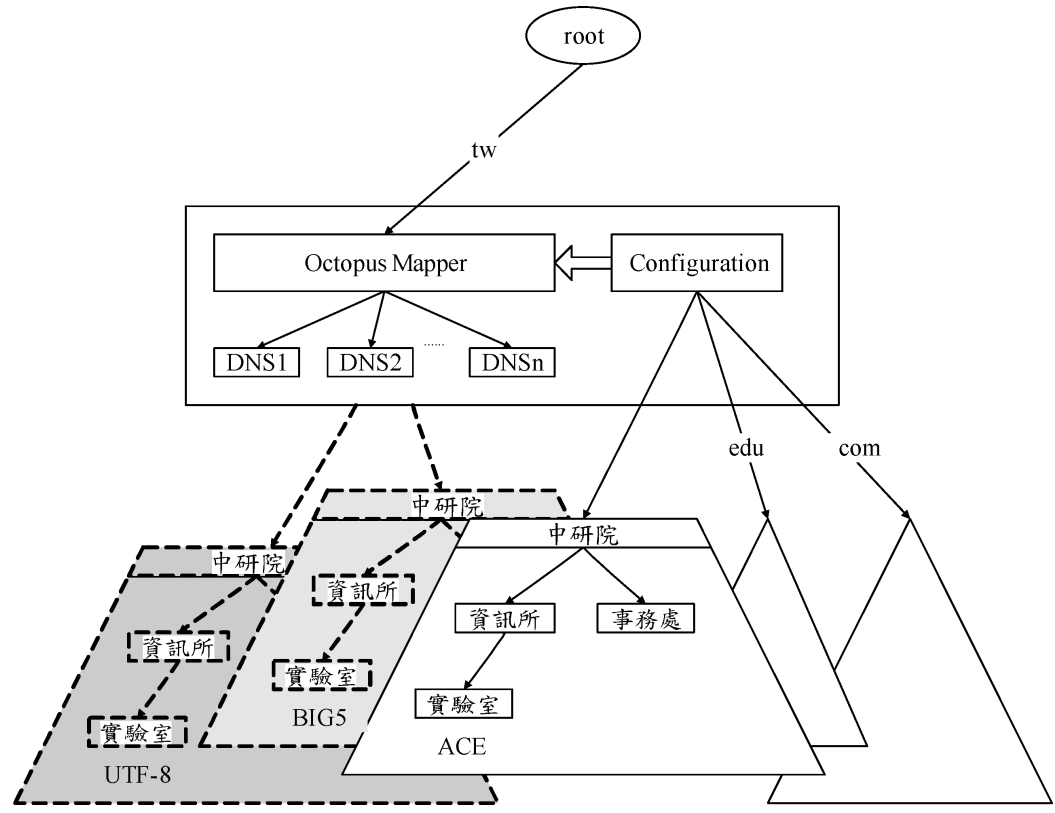

Fig. 4. System architecture of Octopus.

IDN in the QNAME field, 2) forward transcodes the IDN into ACE, 3) passes ACE DNS lookups to $\mathrm{DNS}_{1}, \mathrm{DNS}_{2}$ to $\mathrm{DNS}_{\mathrm{n}}, 4$ ) backward transcodes relevant IDNs, and 5) returns authoritative answers to the remote user.

\subsection{Issues in Designing an IDN Server Proxy}

In this section, we present how we address the issues listed in the previous section in the design of Octopus.

3.1.1 Detection of Encoding. We believe that binary collision prevention can be best accomplished during registration. In other words, a content or service provider that uses Octopus to resolve IDNs must implement a registration protocol to avoid binary collision. However, some zones may not be well managed. In the Octopus Mapper, we use a prioritizing mechanism to handle binary collisions and detect the encoding. When the Octopus Mapper is set up, a priority is assigned to each encoding. Upon receiving a DNS query packet, the Octopus Mapper will try to detect the encoding used to encode the string in the QNAME field. For each encoding, the Octopus Mapper 1) decodes the string into a sequence of code points, 2) performs a code point range check on the sequence, 3) forward transcodes the sequence into an ACE string, and 4) performs a regular DNS lookup for the ACE string. According to the results of these lookups and the priorities of each encoding, the Octopus Mapper determines the encoding used to encode the IDN in the QNAME field. Table I shows the three possible answers Octopus Mapper will give. In case 1, when at least one lookup is positive (successfully resolved) and all other high priority lookups are negative (unsuccessfully resolved), the Octopus Mapper adopts the 
Table I. Priority-Based Handling of Binary Collision

\begin{tabular}{|l|c|c|c|c|c|c|}
\hline Encodings & $\mathrm{E}_{1}$ & $\ldots$ & $\mathrm{E}_{\mathrm{k}}$ & $\ldots$ & $\mathrm{E}_{\mathrm{n}}$ & \multirow{2}{*}{ Answer } \\
\hline Priority & 1 & $\ldots$ & $\mathrm{k}$ & $\ldots$ & $\mathrm{n}$ & $\mathrm{E}_{\mathrm{k}}$ (case 1) \\
\hline \multirow{2}{*}{$\begin{array}{l}\text { Query } \\
\text { Results }\end{array}$} & - & - & + & $\mathrm{X}$ & $\mathrm{X}$ & case 2) \\
\cline { 2 - 7 } & - & - & - & - & - & - (case 3) \\
\cline { 2 - 7 } & - & - & $\mathrm{T}$ & $\mathrm{X}$ & $\mathrm{X}$ & $\mathrm{T}$ (case \\
\hline
\end{tabular}

+: positive, -: negative, $\mathrm{X}$ : don't care, T: timeout.

highest priority encoding that causes a successful lookup. In case 2, when all lookups are negative, the Octopus Mapper returns an unsuccessful response to the remote user. In case 3, when a timeout occurs and all other high priority lookups are negative, the Octopus Mapper discards the query, and a timeout response will be given to the remote user. The prioritizing mechanism ensures that the Octopus IDN System returns a consistent answer even if the domain name servers of ACE subdomain have crashed.

Service providers who adopt Octopus to resolve IDNs may choose the target charsets that are most commonly used by their clients. For example, a service provider who targets providing services to Chinese clients may choose to configure Octopus to handle charsets including BIG5, GB-2312/GBK, UTF8, and punycode [Costello 2003], and so on. On the other hand, a service provider who targets users of mixed Cyrillic and Latin characters may choose to use the proper charsets.

3.1.2 Transcoding Non-ACE and ACE Domain Names. Since ACE is an encoding for Unicode, forward transcodings into ACE should always be successful. If the length of the ACE IDN is longer than the allowed value, the IDN is apparently not existent. However, backward transcoding a relevant IDN into another encoding may fail or generate a domain name string whose length is too long. By default, the Octopus Mapper considers any transcoding failure an unsuccessful lookup. We provide a configurable option so that administrators can manually configure the Octopus Mapper to return relevant IDNs in ACE when the Octopus Mapper cannot successfully backward transcode them.

3.1.3 Routing of a Non-ACE IDN Lookup. In order to route non-ACE IDN lookups to the Octopus Mapper, the Octopus IDN System will tell remote autonomous systems that the Octopus Mapper is authoritatively responsible for non-ACE IDNs subdomains. The value of the NS resource record for each nonACE IDN subdomain is assigned to the IP address of the Octopus Mapper. In addition, the Octopus Mapper will authoritatively answer with a SOA resource record for any non-ACE IDN subdomain, or the Octopus Mapper will be detected and marked as a lame delegation [InterNIC] of that subdomain.

3.1.4 Inference of Domain Name Caching. In order to decrease the possibility that a cache domain name server falsely matches IDNs in its cache, the Octopus Mapper is capable of dynamically adjusting the TTL (Time-To-Live) field of a resource record in the response packet. By making a trade-off between the response time and the possibility of lookup errors, administrators of the Octopus IDN System can reduce the TTLs of resource records associated with error-prone IDNs such as those encoded in BIG5. 
3.1.5 Availability and Scalability. The Octopus Mapper is designed to behave like a true domain name server. Therefore, we can replicate multiple Octopus Mappers with the same method we use to replicate secondary domain name servers in the traditional DNS infrastructure. Thus, the availability of the Octopus IDN System is enhanced. In addition, non-ACE IDN lookups are distributed to multiple Octopus Mappers so that the load can be shared. Since resource consuming ACE IDN lookups are performed by $\mathrm{DNS}_{1}, \mathrm{DNS}_{2}$, or $\mathrm{DNS}_{\mathrm{n}}$, the Octopus Mapper can handle more IDN lookups simultaneously. $\mathrm{DNS}_{1}, \mathrm{DNS}_{2}$, to $\mathrm{DNS}_{\mathrm{n}}$ also provide caches. It is most likely that caches can shorten the time needed for the Octopus Mapper to look up ACE IDNs.

Currently, the Octopus Mapper is capable of handling encodings, including BIG5, GB-2312/GBK, UTF8, and punycode, for Chinese domain names. Since it is designed with modules, Octopus Mapper can easily be adapted to support other encodings. It accepts IDN lookups for all types of resource records specified in Mockapetris [1987], such as A, CNAME, MX, NS, SOA, and PTR, through either a UDP message or a TCP connection. DNS extensions and zone transfers are currently not supportable.

The Octopus Mapper is not able to detect whether the received non-ACE IDN is falsely interpreted. Consequently, it will return an incorrect answer if it receives a vitiated representation of an IDN. We suggest that service providers do not register IDNs that may be falsely interpreted by some platforms.

\section{EXPERIENCES AND DISCUSSIONS}

In July 2002, an experimental Octopus IDN System was installed to manage Chinese domains in the mdns.iis.sinica.edu.tw domain. To evaluate the IDN resolution functionality of Octopus, we entered a total of 10,872 Chinese domain names currently registered in the $t w$ domain. We then used ping on various platforms to evaluate the functionality of Octopus name resolution. Results show that Octopus is effective in providing UTF8 and BIG5 IDN resolution. Some Internet applications, such as Web browsers and servers, perform not only domain name lookups, but also more complicated domain name operations. To study the interoperability of legacy Internet applications when non-ASCII domain names were used, we used Internet Explorer to browse the homepages of 600 sites via their registered Chinese domain names. We also registered into Octopus a total of 105 Chinese domain names each associated with an existing host in the iis.sinica.edu.tw domain. We then performed a series of experiments accessing Internet services (e.g., http, telnet, and ftp) provided by these hosts to further investigate the functional correctness of our IDN service architecture. The architecture functions well for application clients such as telnet and ftp. Here, a client uses the domain name to look up the IP address of the server; however, the client and the server do not have to directly exchange domain name information. Conversely, in applications such as http, the client and the server exchange URLs, and thus domain names extensively. We find that some Web pages cannot be successfully displayed because Web servers or browsers perform more complicated operations over the domain names. In this section, we present our observations, experiment 
Table II. Results of Name Lookup by Applications (ping)

\begin{tabular}{|l|c|c|c|}
\hline Platform & Encodings & Fail in Looking Up & Failure Percentage \\
\hline Windows 98 & BIG5 & 86 & $0.8 \%$ \\
\hline Windows 2000 & UTF8 & 0 & 0 \\
\hline Linux & BIG5 & 86 & $0.8 \%$ \\
\hline FreeBSD & BIG5 & 86 & $0.8 \%$ \\
\hline
\end{tabular}

results, some problems we encountered in providing CDN services, and the solutions.

\subsection{Testing Octopus IDN Resolution Function}

We collected 10,872 Chinese domain names registered under the tw domain. The 10,872 IDNs were registered into Octopus. They were associated with 5,044 CNAME records, 2,540 A records, $25 \mathrm{NS}$ records of 25 subdomains, and 3,263 A records under these subdomains. We first tested the functionality of the Octopus Mapper by using nslookup and dig that have been modified to properly handle non-ASCII domain name strings. As we expected, Octopus provides perfect BIG5 and UTF8 IDN resolution. We then used ping on Windows 98/2000, Linux, and FreeBSD to evaluate the effective IDN resolution provided by Octopus. Results are shown in Table II. Octopus provides perfect name resolution for all UTF8 IDNs. However, 86 BIG5 IDNs are unsuccessfully resolved on the Windows 98, Linux, and FreeBSD. We notice that the three sets of unsuccessfully resolved IDNs are the same. All of the 86 BIG5 IDNs contain an ASCII byte $\backslash$ (backslash) and are therefore falsely interpreted by the resolvers on the three platforms. Octopus cannot transcode the vitiated IDN into ACE and so returns negative answers. In order to prevent the client resolver from falsely modifying the IDN, the user may append an extra \to the Chinese character containing a $\backslash$. By doing so, ping successfully returns the status of the 86 hosts on the three platforms.

\subsection{Experiences in Providing CDN Web Services}

Some applications perform not only domain name lookups, but also more complicated domain name operations. For example, Web servers may have to examine the domain name in order to decide which resource the user wants to access. Web browsers must compute the absolute URLs from relative URLs and domain names. However, most Web servers and browsers are not designed to use Chinese domain names. If those operations on Chinese domain names cause unexpected side effects, the browser would be unable to display the Web pages successfully. A simple evaluation was performed to examine the status of CDN Web services in May 2002. We used Internet Explorer versions 5.0 SP2, $5.0 \mathrm{SP} 2$, and 6.0 SP1, respectively, on Windows 98,2000 , and XP to browse the homepages of 600 sites via Chinese domain names.

600 sites were randomly selected from the $t w$ domain. They are distributed over 174 hosts running different Web servers including among others: Apache 1.3 and 2.0 and Microsoft IIS 3.0, 4.0, and 5.0. Table III shows the statistics of the 174 Web servers, and Table IV shows how the 600 sites are distributed 
Table III. Statistics of Web Servers

\begin{tabular}{|l|l|l|l|l|l|l|l|l|l|l|l|}
\hline & \multicolumn{3}{|c|}{ Apache } & \multicolumn{3}{c|}{ Microsoft IIS } & \multicolumn{3}{c|}{ Netscape-Enterprise } & \multirow{2}{*}{} \\
\cline { 2 - 11 } & 1.3 & 2.0 & others & 4 & 5 & others & 3.6 & 4.0 & others & Others \\
\hline Number of Servers & 51 & 1 & 5 & 10 & 91 & 1 & 4 & 2 & 2 & 7 \\
\hline Percentage & $29.3 \%$ & $0.6 \%$ & $2.9 \%$ & $5.7 \%$ & $52.3 \%$ & $0.6 \%$ & $2.3 \%$ & $1.1 \%$ & $1.1 \%$ & $4.0 \%$ \\
\cline { 2 - 9 } & \multicolumn{3}{|c|}{$32.8 \%$} & \multicolumn{3}{c|}{$58.6 \%$} & \multicolumn{3}{c|}{$4.6 \%$} & $4.0 \%$ \\
\hline
\end{tabular}

Table IV. Distribution of Web Sites

\begin{tabular}{|c|c|c|c|}
\hline $\begin{array}{l}\text { Number of } \\
\text { Servers }\end{array}$ & $\begin{array}{c}\text { Number of Sites Served } \\
\text { by Each Server }\end{array}$ & $\begin{array}{c}\text { Total Number } \\
\text { of Sites }\end{array}$ & Notes \\
\hline 111 & 1 & 111 & 38 Apache 1.3, 49 IIS 5, etc. \\
\hline 35 & 2 & 70 & 5 Apache 1.3, 26 IIS 5, etc. \\
\hline 9 & 3 & 27 & \\
\hline 4 & 4 & 16 & \\
\hline 6 & 5 & 30 & \\
\hline 2 & 7 & 14 & IIS 5 \\
\hline 2 & 8 & 16 & IIS 5 \\
\hline 1 & 10 & 10 & Apache 1.3 \\
\hline 1 & 21 & 21 & Apache 1.3 \\
\hline 1 & 33 & 33 & IIS 5 \\
\hline 1 & 49 & 49 & \\
\hline 1 & 203 & 203 & \\
\hline
\end{tabular}

over these Web servers. Most of the hosts serve one or two sites, and only five hosts serve more than eight sites. Generally speaking, hosts that serve a small number of sites, in fact, serve a single site that is registered with multiple names (aliases). In this case, the Web server does not have to decide which site the browser wants to access, but Web servers that genuinely serve multiple sites must decide which site the browser wants to access. If the server cannot properly handle Chinese domain names or if it is not configured with the necessary Chinese domain name, it will not be able to decide which site the browser wants to access.

We browsed and labeled the 600 homepages into four classes and ten subclasses. Pages in the success class have been successfully displayed by Internet Explorer. Upon further investigation of these pages, we notice that some are hosted on a URL redirection service. In these cases, the browser uses the Chinese domain name to retrieve the page from the URL redirection service. The browser is then redirected to another page that is accessed via a traditional domain name. According to the redirection method, these pages are further categorized into subclasses: pages redirected to another page associated with an ASCII URL, pages refreshed to another page associated with an ASCII URL, and pages containing frames associated with ASCII URLs.

Pages are categorized as a partial success if some of their embedded objects cannot be downloaded or displayed. After examining the sources of these pages, we notice that nondisplayable objects are associated with relative URLs and the browser displays them successfully if absolute URLs are manually entered into the address bar. It is reasonable to assume that browsers may have unexpected problems when using relative URLs and Chinese domain names to retrieve objects. 
Table V. Results of the Homepages from the 600 Sites

\begin{tabular}{|c|c|c|c|c|c|c|c|c|c|c|}
\hline \multicolumn{2}{|c|}{$\begin{array}{ll} & \text { Browsers } \\
\text { Classes }\end{array}$} & \multicolumn{3}{|c|}{$\begin{array}{c}\text { IE } 5.0 \text { on } \\
\text { Windows } 98\end{array}$} & \multicolumn{3}{|c|}{$\begin{array}{c}\text { IE } 5.0 \text { on } \\
\text { Windows } 2000\end{array}$} & \multicolumn{3}{|c|}{$\begin{array}{c}\text { IE } 6.0 \text { on } \\
\text { Windows XP }\end{array}$} \\
\hline \multirow{4}{*}{ Success } & No redirection & 236 & $39.33 \%$ & \multirow{4}{*}{$57.67 \%$} & 231 & $38.50 \%$ & \multirow{4}{*}{$57.33 \%$} & 300 & $50.00 \%$ & \multirow{4}{*}{$69.00 \%$} \\
\hline & $\begin{array}{l}\text { Pages redirected to } \\
\text { another page } \\
\text { associated with } \\
\text { an ASCII URL }\end{array}$ & 30 & $5.00 \%$ & & 32 & $5.33 \%$ & & 33 & $5.50 \%$ & \\
\hline & $\begin{array}{l}\text { Pages refreshed to } \\
\text { another page } \\
\text { associated with } \\
\text { an ASCII URL }\end{array}$ & 10 & $1.67 \%$ & & 10 & $1.67 \%$ & & 10 & $1.67 \%$ & \\
\hline & $\begin{array}{l}\text { Pages containing } \\
\text { frames } \\
\text { associated with } \\
\text { ASCII URLs }\end{array}$ & 70 & $11.67 \%$ & & 71 & $11.83 \%$ & & 71 & $11.83 \%$ & \\
\hline \multirow{2}{*}{$\begin{array}{l}\text { Partial } \\
\text { Success }\end{array}$} & $\begin{array}{l}\text { Relative frame not } \\
\text { found }\end{array}$ & 72 & $12.00 \%$ & & 72 & $12.00 \%$ & \multirow[b]{2}{*}{$14.00 \%$} & 6 & $1.00 \%$ & \multirow[b]{2}{*}{$2.33 \%$} \\
\hline & $\begin{array}{l}\text { Relative object not } \\
\text { found }\end{array}$ & 11 & $1.83 \%$ & $13.83 \%$ & 12 & $2.00 \%$ & & 8 & $1.33 \%$ & \\
\hline \multirow[t]{2}{*}{ Fail } & $\begin{array}{l}\text { Error code: } 400 \text { or } \\
\quad 404\end{array}$ & 115 & $19.17 \%$ & \multirow{2}{*}{$26.67 \%$} & 117 & $19.50 \%$ & \multirow{2}{*}{$26.83 \%$} & 117 & $19.50 \%$ & \multirow[t]{2}{*}{$26.83 \%$} \\
\hline & Page not found & 45 & $7.50 \%$ & & 44 & $7.33 \%$ & & 44 & $7.33 \%$ & \\
\hline \multirow[b]{2}{*}{ Unknown } & Under construction & 7 & $1.17 \%$ & \multirow[b]{2}{*}{$1.83 \%$} & 7 & $1.17 \%$ & \multirow{2}{*}{$1.83 \%$} & 7 & $1.17 \%$ & \multirow[b]{2}{*}{$1.83 \%$} \\
\hline & Prohibited & 4 & $0.67 \%$ & & 4 & $0.67 \%$ & & 4 & $0.67 \%$ & \\
\hline
\end{tabular}

Pages are categorized as a failure either because the browser receives an error code (such as 400 (bad request) or 404 (not found)) in the response header [Berners-Lee 1996; Fielding et al. 1997], or the server returns a page that tells the user that it cannot find the requested object. As we described, it is likely that the server cannot handle Chinese domain names properly or the server is not well configured.

Finally, there are eleven pages categorized as uncertain, including seven under construction pages and four prohibited pages. Without further information, we cannot discern whether these pages are indeed under construction or prohibited or if they are default pages returned because the Web server cannot handle Chinese domain names properly.

Table V shows the results from the 600 sites' homepages, and Figure 5 gives the aggregated results.

To further investigate the side effects of using Chinese domain names, we registered into Octopus a total of 105 Chinese domain names, each associated with an existing host in the iis.sinica.edu.tw domain. We then performed a series of experiments accessing Internet services (e.g., http, telnet, and ftp) provided by these hosts and tried to eliminate the side effects.

As we expected, for Internet protocols such as telnet and ftp, Octopus IDN service architecture functions well. However, we find that browsers could not successfully display some pages when we used the registered Chinese domain names to access these Web sites.

Following, we introduce three types of errors observed, and explain how we deal with them. 


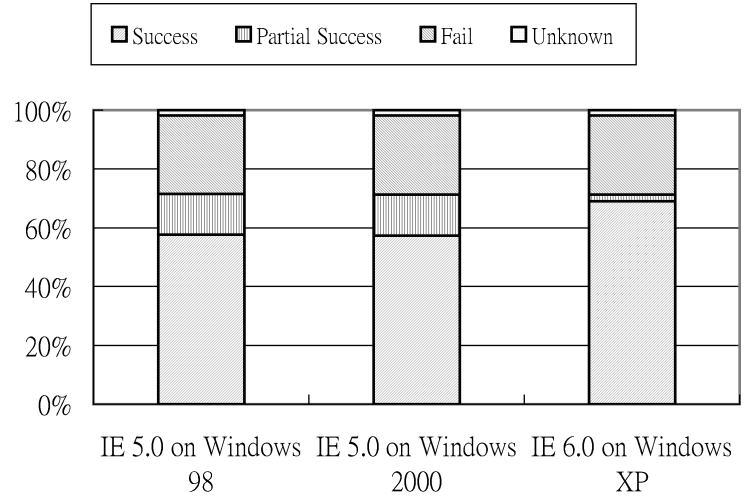

Fig. 5. Aggregated results from the homepages of the 600 sites.

4.2.1 Browser Errors. ASCII characters may occur as the second byte in many double-byte charset encodings such as in BIG5. If an IDN encoded in one of these encodings contains ASCII characters with special meanings, browsers may have problems parsing the URL correctly. If the IDN contains character @, some versions of Internet Explorer (earlier than 5.5) will falsely recognize the @ as a separator and therefore decouple the IDN into two parts, username and hostname. As a result, the browser is unable to retrieve the object via the fragmented IDN. There are tricks to fool Internet Explorer, for example, one can place an @ before the IDN. However, the browser eventually must be patched in order to correctly recognize IDNs with different encodings.

We also notice that some pages are partially rendered because embedded objects are not retrieved. To understand why Internet Explorer cannot retrieve these objects, we performed a series of experiments. We find that Internet Explorer has unexpected problems using relative URLs to retrieve embedded objects if the page does not specify the charset correctly. If the page correctly specifies its charset, both Internet Explorer version 5.0 and 6.0 will successfully display these objects. If the page does not specify the charset, Internet Explorer 6.0 can retrieve these objects via the IDN and relative URLs, but Internet Explorer 5.0 cannot. If the page specifies the charset as something else, both versions of Internet Explorer will fail in retrieving these objects. Authors of Web pages can help prevent this problem by specifying the correct charset or using absolute URLs.

4.2.2 Virtual Host Server Errors. Upon receiving a request, a Web server should discover the object the user wants to access. If a server hosts only one Web site, it might not have to examine the Host field in the request header. If a server hosts more than one Web site, each with a different domain name, it should examine the Host field in the request header to discover the site the user wants to access. Some servers will reject non-ASCII domain names and return a bad request message. If a Web server accepts non-ASCII domain names, it will search its configuration to find a match. If it cannot find a match, it returns an error code in the response header or a default page to tell the user that it 
cannot locate the requested object. This is the most likely reason for such pages to be classed as failure.

We notice that Microsoft IIS and Apache Web servers will not examine the domain name in the request header if these servers are not configured to host more than one Web site. In this case, the browser can download and display the objects on these servers. If the Web server administrators want to host more than one Web site accessed via IDNs, they must configure the IDNs into their Web servers. Microsoft IIS Web servers accept non-ASCII domain names. However, some early versions of Apache do not. The administrators must patch their Apache Web servers in order to configure non-ASCII domain names. For Web servers that cannot be configured with non-ASCII domain names and do not have patches, the administrators can consider subscribing a URL redirection service that will redirect their IDN homepages to the homepages that are accessed via traditional domain names.

4.2.3 Application Server Errors. Among the many server scripting languages and tools for Web development, some are not safe to incorporate with IDNs. As an example, a Web page author may want to redirect an http request to another URL. When using ASP (Active Server Pages), he or she may invoke the method Response.Redirect with the target URL as an argument. However, this method invokes the function URLencode() which encodes the entire URL by substitution "\%hh"-formatted escaped sequences for non-ASCII characters [Berners-Lee et al. 1994, 1998]. If the browser does not know the IDN is thus encoded in this format, the redirection will fail. Authors who use JSP (Java Server Pages) may encounter a similar condition. JSP always considers a nonASCII domain name as a UTF8 string. Each non-UTF8-compliant part will be replaced with a three-octet binary sequence 'EF BF BD' (in hexadecimal). ${ }^{4}$ Authors who use ASP or JSP should develop a new procedure to redirect requests to IDN URLs. For example, they can use the refresh method that will be described in the following section.

\subsection{IDN Web Hosting}

As we have described, Octopus can effectively provide IDN resolution. However, some problems frustrate user attempts to use IDNs in applications. For example, Web server administrators do not configure the Web servers correctly. Writing FAQ (frequent asked questions) documents for using IDNs in Web services can help Web server administrators and Web page authors understand how to deal with these problems. Another way for name holders to activate their IDN homepages is to subscribe to an IDN Web hosting service that will redirect IDN http requests to pages that are accessed via traditional domain names. All the name holder has to do is 1) subscribe to an IDN Web hosting service and 2) register the IDN associated with the IP address of the redirection server of the IDN Web hosting service. When users use the IDN to access the name holder's homepage, after looking up the IP address, the request will be sent to

${ }^{4}$ The sequence 'EF BF BD' (in hexadecimal) is the UTF8 representation of a Unicode character $\mathrm{U}+\mathrm{FFFD}$, which represents a replacement. 


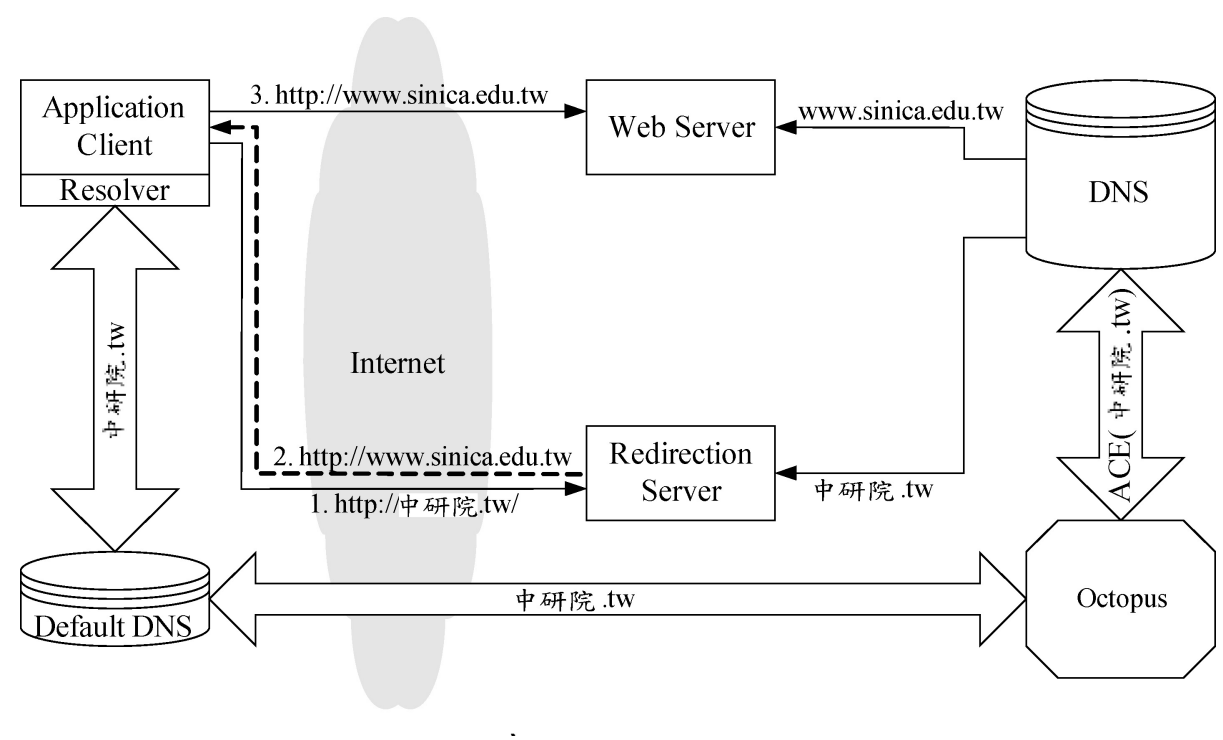

Fig. 6. Redirect http://中研院.tw to http://www.sinica.edu.tw.

the redirection server of the IDN Web hosting service. The redirection server processes the request and then redirects the request to the page specified by the name holder. IDN Web hosting service is a simpler and faster way for name holders to activate their IDN homepages.

We set up a redirection server to investigate IDN Web hosting services and redirection of IDN http requests. Figure 6 depicts the scenario. The name holder registers the IDN associated with the IP address of the redirection server into Octopus, and also enters the IDN and corresponding traditional domain name of the designated page into the database of the redirection server. When browsers use the IDN to look up the IP address, Octopus will return the IP address of the redirection server. Then the browsers will open an http connection to the redirection server and send the request. The redirection server accepts the request, detects the encoding of the IDN, and searches its database for the corresponding traditional domain name of the designated Web server. Finally, the redirection server returns a redirection message to the client so the client can retrieve the resource using the traditional domain name.

There are many applicable redirection techniques. The first is to return an http response with a redirection 3xx status code and the target URL found in the database. A similar technique is to refresh. For example, the tag $<$ meta HTTP-EQUIV="refresh" Content="1; URL=http://www.sinica.edu.tw"> will make the browser refresh the current page to http://www.sinica.edu.tw. The third technique is to use frames. The returned page contains a full-size frame that is associated with the target URL. The advantage of the third technique is that the URL, and thus the IDN, in the address bar will not change.

We set up a generic PC running a Linux operating system with an Apache 1.3 Web server as the redirection server in our IDN Web hosting service. The Web server is patched to accept non-ASCII domain names and configured to host 
Table VI. Cost of Modifying Software to Provide IDN Services

\begin{tabular}{|c|c|c|c|c|}
\hline \multicolumn{2}{|r|}{ Architecture } & \multirow[b]{2}{*}{ IDNA } & \multirow[b]{2}{*}{ IDN Proxy } & \multirow[b]{2}{*}{ IDN Server Proxy } \\
\hline \multicolumn{2}{|l|}{ Relevant Software } & & & \\
\hline \multirow{6}{*}{ DNS layer } & Resolver & No & No & No \\
\hline & IDN proxy & - & \multirow[t]{2}{*}{ All ISPs } & - \\
\hline & Default DNS servers & No & & No \\
\hline & $\begin{array}{l}\text { Domain name server of } \\
\text { Name Holders }\end{array}$ & No & No & No \\
\hline & $\begin{array}{l}\text { Domain name server of } \\
\text { Others }\end{array}$ & No & No & No \\
\hline & IDN server proxy & - & - & Service Providers \\
\hline \multirow{2}{*}{ Application layer } & $\begin{array}{l}\text { Legacy applications that } \\
\text { can handle non-AE } \\
\text { IDNs }\end{array}$ & All & No & No \\
\hline & $\begin{array}{l}\text { Legacy applications that } \\
\text { can not handle } \\
\text { non-ACE IDNs }\end{array}$ & All & All & All \\
\hline
\end{tabular}

multiple sites. When an http request arrives, the server tries to find a match from the configuration. If the server cannot find a match, it executes a script to look up the requested IDN in its database and generates a page that contains a frame associated with the designated URL.

We register a total of 75 IDNs for 20 existing Web sites under the iis.sinica.edu.tw domain into Octopus. Each of the 75 IDNs is associated with the IP address of the redirection server. We then enter into the database of the redirection server the corresponding traditional domain names for each of the 75 IDNs. We use Internet Explorer and Netscape on Windows 98, 2000 and XP, Linux, and FreeBSD to access these IDN homepages. Results show that our IDN Web hosting service successfully enables users to use IDNs to access Web sites.

\section{CONCLUSION}

Although most Internet protocols recommend that Internet applications use ASCII domain names in the hostname fields, many applications allow users to use non-ASCII domain names. On the other hand, Internet RFCs on URL and URL recommend that applications substitute "\%hh"-formatted escaped sequences for non-ASCII characters. This somewhat contradicts the new IETF RFCs on IDNA. Consequently, domain name servers and application servers may receive IDNs in different encodings.

Table VI summarizes the cost of modifying relevant software to provide IDN services. IDNA recommends that Internet applications convert a Unicode IDN into an ACE string and use this ACE string to access the Internet host. However, some of the domain names embedded in multilingual content do not have any charset tag and therefore cannot be converted into ACE strings. IDNA uses the existing DNS infrastructure without any modification. However, it does require that an IDNA-compliant be integrated into every Internet applications. IDN proxies can provide IDN resolution for users of legacy Internet applications. However, all ISPs are required to set up IDN proxies. There is an issue of 
consistency between all IDN proxies. As well, IDN proxies may have problems in recognizing all of the different kinds of encodings that users or autonomous systems might use.

We propose a new architecture, IDN server proxy, which provides transparent IDN resolution on the domain name server side. In our architecture, ACE IDNs are stored in the domain name servers of the name holders so traditional domain name servers can be used. The service providers can employ Octopus to facilitate these domain name servers to provide non-ACE IDN resolution. This allows users of legacy Internet applications the capability of handling non-ASCII domain names to access Internet hosts.

Based on the implementation of Octopus, we started a CDN service trial to study the interoperability of legacy Internet applications. Our studies help service providers understand potential problems when non-ASCII domain names are used and best common practice at this stage. Experiment results show that if Web server administrators and Web page authors were more careful in regard to IDNs, many problems that occur in current IDN Web services could be avoided. In software practice, communication protocols require more investigation between application servers and clients when different charset encodings are involved. The experiences also give some guidelines for software developers in developing IDNA-compliant Internet applications. Other future research issues include IDNs in email, character variant problems, and so on. Character variants, such as those in the Han character set, can generate equivalent IDNs and thus cause serious consumer protection problems. The current trend for handling character variant problems is to register equivalent IDNs [Hoffman 2003; Konishi 2004]. However, there is an issue of scalability when the number of equivalent IDNs is too large. Internationalized domain names should be further localized to reflect the language issues of individual cultures.

\section{ACKNOWLEDGMENTS}

Many thanks to June-Yen Huang and Zhi-Zhueng Huang, at the Institute of Information Science, Academia Sinica, for their help in the development of the Octopus IDN System.

\section{REFERENCES}

Berners-Lee, T., Fielding, R., And Frystyk, H. 1996. Hypertext Transfer Protocol-HTTP/1.0. RFC 1945.

Berners-Lee, T., Fielding, R., ANd Masinter, L. 1998. Uniform Resource Identifiers (URI): Generic Syntax. RFC 2396.

Berners-Lee, T., Masinter, L., and McCahill, M. 1994. Uniform Resource Locators (URL). RFC 1738.

Chung, E. And Leung, D. 2002. Internationalized Domain Name Transition (IDNX). Internet Draft.

Costello, A. M. 2003. Punycode: A Bootstring encoding of Unicode for IDNA. RFC 3492.

Faltstrom, P., Hoffman, P., ANd Costello, A. M. 2003. Internationalizing Domain Names in Applications (IDNA). RFC 3490.

Fielding, R., Gettys, J., Mogul, J., Frystyk, H., and Berners-Lee, T. 1997. Hypertext Transfer Protocol-HTTP/1.1. RFC 2068.

Hoffman, P. 2003. A Method for Registering Internationalized Domain Names. Internet Draft. 
Hoffman, P. And Blanchet, M. 2002. Preparation of Internationalized Strings (stringprep). RFC 3454.

Hoffman, P. and Blanchet, M. 2003. Nameprep: A Stringprep Profile for Internationalized Domain Names. RFC 3491.

INTERNIC. InterNIC Name Delegation Policy. www.internic.net.

ISO/IEC 10646-1:2000(E). I nternational Standard-Information Technology. Universal Multiple-Octet Coded Character Set (UCS).

JPNIC. Internationalized/Multilingual Domain Name Tool Kit (idnkit/mDNkit). http://www.nic. ad.jp/en/idn/index.html.

Konishi, K., Huang, K., Qian, H., AND KO, Y. 2004. Joint Engineering Team (JET) Guidelines for Internationalized Domain Names (IDN) Registration and Administration for Chinese, Japanese, and Korean. RFC 3743.

Mockapetris, P. 1987. Domain Names-Concepts and Facilities (RFC 1034) and Domain NamesImplementation and Specification (RFC 1035). STD 13.

The Unicode Consortium. The Unicode Standard. http://www.unicode.org/.

YeRgEAU, F. 1998. UTF-8: A Transformation Format of ISO 10646. RFC 2279.

Received September 2003; revised August 2004; accepted December 2004 